\title{
Diphtheric Myocarditis: A Resurgence in Urban Bangalore, India
}

\section{Bharath Kumar Reddy*, Basavaraja GV and Govindaraju M}

Department of Pediatric Intensive Care, Indira Gandhi Institute of Child Health, Bangalore, India

\section{Introduction}

The introduction of the Expanded Program on Immunization (EPI) by the World Health Organization (WHO) in 1978 and the Universal Immunization Program (UIP) in 1985 had resulted in the virtual elimination of diphtheria in many developed countries. Other than the major resurgence noted in 1989, a decreasing trend has continued in the world. Small epidemics have occurred in South East Asia, India, Pakistan and South America, strongly emphasizing the need for maintaining full immunization coverage, failing which there stands an increased chance that the disease may return [1].

In the prevaccination era, diphtheria was a leading cause of childhood mortality with $15 \%$ children being symptomatic and the rest subclinical, along with naturally acquired immunity [2]. Diphtheria in India has shown to be endemic in certain areas [3]. The total number of cases of diphtheria in India during 1980 was about 39,231, which reduced to 3094 cases in 2000 and then increased to 5725 cases in 2005 [4]. As per a report of WHO/UNICEF, 2004 the estimated DPT-3 coverage among children in India has dropped from 71\% in 1990-1995 to $64 \%$ every year from 2000 to 2004 [5]. The figures quoted above may possibly be a gross underestimate due to lack of a good surveillance system and facilities for microbiologic diagnosis [6]. In a case series of 616 cases over a period of 5 years (1989-1993) from West Bengal, the diagnosis of diphtheria was clinical, microbiologic confirmation being available in only $7 \%$. The series demonstrated an age shift, as $40 \%$ of the cases occurred above the age of 5 years. In 2008, India contributed 6081 (86.66\%) of the 7017 diphtheria cases reported globally [7]. Data on the incidence of diphtheria in urban Bangalore is minimal and insufficient. The annual incidence reported in an urban city in the neighboring state of Andhra Pradesh shows that the incidence of diphtheria in Hyderabad had increased from $11 / 100,000$ to $23 / 100,000$ from 2003 through 2006 [8].

Vaccine induced immunity does not last for life and various serological studies conducted in developed countries have shown adults being potentially susceptible, especially with incomplete immunization. Low levels of adult immunity and gradual increase in number of children not receiving three doses of DPT vaccine poses an imminent threat to resurgence. All the epidemics have been associated classically with toxin-producing Corynebacterium diphtheria, with the production of an exotoxin.

The disease is subdivided into three stages - early, late and severe. In the early stages, manifestations are localized to either the skin or the upper respiratory tract which can lead on to the severe stage associated with toxic circulatory collapse, edema of the neck, skin petechiae and acute renal failure. The late stages can involve cardiac and neurological involvement such as myocarditis, respiratory paralysis and limb paralysis. All stages are associated with a pseudomembrane formation which bleeds on removal. Cardiac involvement with arrhythmias occurs in one-quarter of patients who have late and severe disease, increasing the mortality to three to four times.

\section{Case Report}

We hereby describe three cases of diphtheria who presented to us within the past year, all of whom developed the dreaded toxic myocarditis leading to ventricular arrhythmias and death. All three children presented with fever since one week along with a noisy breathing and decreased vocalization by the 5 th -6 th day of illness. Two of them were unimmunized and had not received even a single dose of DPT vaccine inspite of living in urban Bangalore. On clinical examination, children had a toxic look with a submandibular swelling, with one child having significant cervical lymphadenopathy involving upper, middle and lower cervical lymph nodes. The vitals at presentation were not stable with all children having an associated tachypnoea and decreased saturation in room air. Examination of the oral cavity revealed a grayish white patch involving both the tonsils, anterior and posterior tonsillar pillars. On indirect laryngoscopy the membrane was found to be extending to the epiglottis, pharygoepiglottic fold and aryepiglottic fold in all three children. The membrane on attempt to removal had spots of bleeding. Routine investigations revealed a neutrophilic leucocytosis. Swabs were taken from the throat and nose of all children and sent for Grams stain, Alberts stain and aerobic/ anaerobic culture and sensitivity. In only one child did the Gram's stain revealed polymorphonuclear leucocytes and Gram positive slender bacilli, which on Alberts stain were found to morphologically resemble Corynebacterium Diphtheriae (Table 1).

The children were immediately started on Penicillin and were given anti-diphtheric serum. The progression of the disease process was similar in all three children. Within 24 hours, they developed a state of shock with tachycardia, prolonged capillary refill and feeble pulses.

\begin{tabular}{|c|c|c|c|}
\hline & $\begin{array}{c}\text { Case 1 (June } \\
\text { 2012) }\end{array}$ & Case 2 (July 2012) & Case 3 (July 2012) \\
\hline Age (years) & 8 & 4 & 4 \\
\hline Gender & Female & Female & Female \\
\hline Residence & Bangalore (Urban) & Bangalore (urban) & Bangalore (urban) \\
\hline $\begin{array}{c}\text { Doses of DPT } \\
\text { received }\end{array}$ & Unimmunized & Unimmunized & 3 doses in 1st year \\
\hline $\begin{array}{c}\text { Pseudomembrane } \\
\text { Stridor }\end{array}$ & ++ & ++ & ++ \\
\hline $\begin{array}{c}\text { Submandibular } \\
\text { edema }\end{array}$ & ++ & ++ & - \\
\hline Myocarditis & ++ & + & + \\
\hline Total counts & 21,200 & 25,900 & ++ \\
\hline Alberts Smear & Negative & Negative & Positive \\
\hline Culture & Negative & Negative & Negative \\
\hline
\end{tabular}

Table 1: Characteristics of children with Diphtheria

*Corresponding author: Bharath Kumar Reddy, Department of Pediatric Intensive Care, Indira Gandhi Institute of Child Health, Bangalore, India, E-mail: bharathreddykr@yahoo.co.in

Received April 29, 2013; Accepted May 13, 2013; Published May 20, 2013

Citation: Reddy BK, Basavaraja GV, Govindaraju M (2013) Diphtheric Myocarditis: A Resurgence in Urban Bangalore, India. J Gen Pract 1: 104. doi: 10.4172/23299126.1000104

Copyright: $\odot 2013$ Reddy BK, et al. This is an open-access article distributed under the terms of the Creative Commons Attribution License, which permits unrestricted use, distribution, and reproduction in any medium, provided the original author and source are credited. 


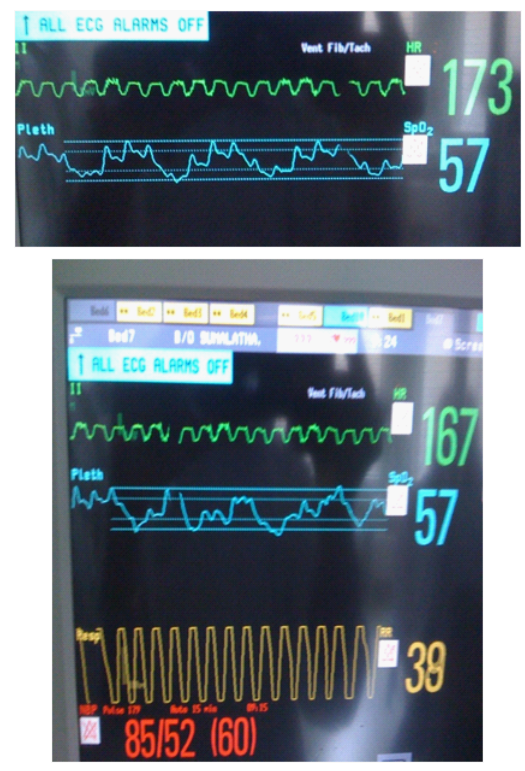

Figure 1: Wide QRS complexes suggestive of ventricular tachycardia with hemodynamic instability.

There was also an associated increase in the respiratory distress. They were ventilated as per the hospital protocol and started on ionotropic support with dobutamine. Over a period of few hours they developed severe disproportionate tachycardia, weak peripheral pulses and muffling of heart sounds. An ECG was done which revealed low voltage complexes with an absence of $\mathrm{p}$ waves, suggestive of a myocarditis. All children were given the 60,000 Units of ADS as the features of myocarditis were established. Inspite of ADS and ionotropic support, they developed wide QRS complexes, suggestive of a ventricular tachycardia. For the immediate management, IV lignocaine was given followed by an amiodarone infusion. The ventricular tachycardia rhythm reverted to a sinus tachycardia rhythm for a short duration of time, which however was followed by a progression to ventricular fibrillation and cardiac arrest. None of the three children could be revived inspite of all resuscitative efforts (Figure 1).

\section{Discussion}

Myocarditis is clinical syndrome characterized by inflammation of myocytes resulting from infectious, toxic, and autoimmune etiologies. Worldwide, the most common bacterial cause is diphtheria. Children present with features ranging from a non specific fatigue and malaise to congestive cardiac failure. Other symptoms include fever, dyspnoea, chest pain and palpitations. Clinical signs include a tachycardia which is disproportionate to the fever with signs of ventricular dysfunction such as jugular venous distention, basilar crackles, ascites, peripheral edema and S3 or a summation gallop.

In one important study [9] conducted in Chandigarh, North India, it was shown that the mean interval between the onset of symptoms and myocarditis was 6.5 days. The incidence of myocarditis noted was $66.6 \%$ which was higher than previously described [10]; $62.5 \%$ of these children presented with conduction abnormalities and cardiogenic shock; $15.6 \%$ had arrhythmias and $3.1 \%$ congestive cardiac failure. The arrhythmias noted included bundle branch block (BBB) tachyarrhythmias (ventricular tachycardia, supraventricular and junctional tachycardia). A univariate analysis revealed that inadequate immunity, longer duration of bull neck and delayed administration of ADS were associated with development of myocarditis. $78.1 \%$ of the children with diphtheric myocarditis died with cardiogenic shock contributing to the predominant cause of mortality.

The importance of childhood booster immunization is elaborated from the Russian epidemic. Disease rates were very high in children aged 7-10 years, with a shift in the age-based incidence. This is attributed to the lack of a booster dose which was not received by these children. Clinical diagnosis should be given more importance than microbiological confirmation rate which is low as observed by Ray et al. [11]. The reasons attributed for low microbiological positivity may be due to administration of antibiotics to the patients in the private sector before admission in the hospital. Serum specific antibody against diphtheria is not easily available for use in the clinical setting in India and thus could not be performed in these children. Overall mortality was seen in $73 \%$ of patients, which may be due to late or missed diagnosis, non-availability of antidiphtheritic serum and low index of clinical suspicion [12]. Hence a high index of suspicion followed by early intervention is required in these children. Inspite of the Albert's stain being negative, the presence of the classical pseudomembrane in a toxic looking child, should enable a clinician to suspect this grave diagnosis in endemic places like India.

The need for the easy availability of the anti-diphtheric serum should be emphasized near the government by avid reporting of diphtheria cases and documentation of the same. Immunization coverage of children against diphtheria is far from satisfactory and is on a decline since the past few years. Efforts to increase both primary and booster immunization should be emphasized. Serologic studies to monitor and detect this transition in immune status in adults are thus required. A good surveillance system thus needs to be established to detect an outbreak right at the outset.

\section{References}

1. Efstratiou A, George RC (1996) Microbiology and Epidemiiology of diphtheria Rev Med Microbiol 7: 31-42.

2. Vitek CR, Wharton M (1998) Diphtheria in the former Soviet Union: reemergence of a pandemic disease. Emerg Infect Dis 4: 539-550.

3. Patel UV, Patel BH, Bhavsar BS, Dabhi HM, Doshi SK (2004) A retrospective study of diphtheria cases, Rajkot, Gujarat. Indian J Community Med 24: 161163.

4. WHO. Immunization surveillance, assessment and monitoring. Immunization Profile - India. 2006.

5. WHO/UNICEF Joint Reporting Form (JRF), India EPI Fact Sheet, 2004.

6. Vitek C, Wenger J (1998) Diphtheria. Bull World Health Organ 76 Suppl 2: 129-130.

7. WHO. Vaccine preventable disease monitoring system, global summary Available romhttp://www.who.int/vaccine documents/Global summary/Global summary pdf.

8. Bitragunta S, Murhekar MV, Hutin YJ, Penumur PP, Gupte MD (2008) Persistence of diphtheria, Hyderabad, India, 2003-2006. Emerg Infect Dis 14: 1144-1146.

9. Jayashree M, Shruthi N, Singhi S (2006) Predictors of outcome in patients with diphtheria receiving intensive care. Indian Pediatr 43: 155-160.

10. Havaldar PV, Patil VD, Siddibhavi BM, Sankpal MN, Jagadish (1989) Fulminant diptheretic myocarditis. Indian Heart J 41: 265-269.

11. Ray SK, Das Gupta S, Saha I (1998) A report of diphtheria surveillance from a rural medical college hospital. J Indian Med Assoc 96: 236-238.

12. Khan N, Shastri J, Aigal U, Doctor B (2007) Resurgence of diphtheria in the vaccination era. Indian J Med Microbiol 25: 434 